\title{
Production and properties of haemolysins from clinical isolates of the Proteeae
}

\author{
B. W. SENIOR and C. HUGHES*
}

Department of Medical Microbiology, Dundee University Medical School, Ninewells Hospital, Dundee DD1 $9 S Y$ and "Microbiology Division, Department of Pathology, Cambridge University, Cambridge CB2 1OP

\begin{abstract}
Summary. A collection of 198 clinical isolates of strains belonging to the tribe Proteeae was examined for haemolytic activity on blood agar and in Brain Heart Infusion Broth. The strains were of diverse bacteriocin and O-antigenic types and from a wide variety of sources. They included representatives of all species of Morganella, Proteus and Providencia. Approximately half of the $M$. morgani strains were haemolytic on blood agar. This activity was not associated with any particular bacteriocin type. The haemolysin was also produced during exponential growth in broth and was thermolabile and calcium dependent. All $P$. mirabilis strains and some $P$. vulgaris strains were non-haemolytic on blood agar. However, most strains of the Proteus spp., irrespective of their bacteriocin and antigenic type, produced, over a short period during exponential growth in broth, a heat-stable, cell-associated calcium-independent haemolysin. A smaller proportion of $P$. vulgaris and $P$. penneri strains produced, in addition, a thermolabile, calcium-dependent haemolysin which was associated with the formation of large haemolytic zones on blood agar. The relationship of these haemolysins to Escherichia coli haemolysin and their possible role in virulence is discussed. Haemolysin production was not found in any of the 74 strains of four species of Providencia.
\end{abstract}

\section{Introduction}

An association between virulence for the urinary tract and haemolysin production in Escherichia coli was first reported by Dudgeon et al. (1921) and subsequently confirmed by others in epidemiological surveys (Cooke and Ewins, 1975; Brooks et al., 1980; Green and Thomas, 1981; Hacker et al., $1983 a$; Hughes et al., 1983). The isolation of haemolysin genes from $E$. coli and their transfer to avirulent strains provided unequivocal evidence that haemolysin production contributes to the virulence of $E$. coli (Welch et al., 1981; Hacker et $a l ., 1983 b$ ).

Proteus mirabilis is another major cause of chronic and recurrent urinary-tract infections and is even found more frequently than $E$. coli in infections of the elderly (Senior, 1979) and young boys (Bergström, 1972; Hallett et al., 1976; Khan et al., 1978). However, other members of the tribe Proteeae are rarely associated with urinary-tract infections and some are more often associated with diarrhoea (Senior, 1979, 1983; Senior and Leslie, 1986). Since Peerbooms et al. (1983) have shown that the

Received 8 Dec. 1986; revised version accepted 24 Feb. 1987. haemolysins produced by $P$. mirabilis influence the virulence of this organism for the mouse, we have investigated the frequency and characteristics of haemolysin production in clinical isolates of this and all other species in the Proteeae to see if the virulence of $P$. mirabilis for the urinary tract is likely to be similarly linked to haemolysin production.

\section{Materials and methods}

\section{Bacterial strains}

A total of 198 strains of eight species belonging to the tribe Proteeae was examined. The strains were selected to represent as wide a variety as possible with regard to type, clinical source, and place of isolation. Most strains were isolated from clinical specimens from unrelated individuals. All strains were purified and stored on nutrient-agar slopes in 7-ml screw-capped bottles at $4^{\circ} \mathrm{C}$.

\section{Media}

Nutrient broth was from Oxoid (CM 67) and Brain Heart Infusion Broth (BHI) from BBL (11059). Each was sterilised by autoclaving at $121^{\circ} \mathrm{C}$ for $15 \mathrm{~min}$. Blood agar was made from Blood Agar Base (Oxoid, CM 55), 
(sometimes supplemented with Bacteriological Agar No. 1 (Oxoid L 11), to give a final total agar concentration of $3 \% \mathrm{w} / \mathrm{v})$. After sterilisation as above, horse red-blood cells (Oxoid SR50) were added to give a final concentration of $5 \% \mathrm{v} / \mathrm{v}$. Tryptophan deaminase agar medium, urea/indole medium, ornithine decarboxylase $/ \mathrm{H}_{2} \mathrm{~S}$ medium and peptone-water sugar media were prepared and sterilised as previously described (Senior and Leslie, 1986). Saline was $0.85 \% \mathrm{NaCl}$ in water.

\section{Speciation of strains}

Strains were identified according to the scheme described by Senior and Leslie (1986).

\section{Serotyping of strains}

Strains were serotyped by the method of Larsson and Olling (1977).

\section{Bacteriocin typing of strains}

Strains of Proteus spp. were typed by their ability to produce (P) bacteriocins (proticines) and also by their sensitivity (S) to proticines- $\mathrm{P} / \mathrm{S}$ typing-according to the method of Senior (1977) and Senior and Larsson (1983). $M$. morgani strains were similarly typed for bacteriocin (morganocin) production and sensitivity (Senior, 1987).

\section{Examination of strains for haemolysin production}

Past studies on haemolysin production by strains of Proteus have given conflicting results (Wenner and Rettger, 1919; Taylor, 1928; Yacob, 1932) because of variations in the type of red-blood cells and culture media used and in the assay conditions (Kotelko et al., 1983; Peerbooms et al., 1983). Waste products of metabolism may also cause haemolysis. To minimise the effect of the latter, shorter incubation periods were used than those of others (Taylor, 1928; Kotelko et al., 1983) and the following procedures adopted as standard. A fresh nutrient-broth culture of each strain was diluted in saline and plated out on blood agar to give rise to well-isolated colonies. Proteus strains that swarmed were plated on blood agar containing agar $3 \% \mathrm{w} / \mathrm{v}$ to obtain discrete colonies. After incubation for $16 \mathrm{~h}$ at $37^{\circ} \mathrm{C}$, plates were immediately examined under good illumination. Those strains with which a zone of lysed red blood cells surrounded each well-isolated colony when the background culture medium remained unchanged were regarded as haemolytic.

In addition, $0.1 \mathrm{ml}$ of an overnight nutrient-broth culture of each strain was added to $10 \mathrm{ml}$ of BHI broth and incubated with shaking at $37^{\circ} \mathrm{C}$. At intervals during growth (measured as absorbance at $550 \mathrm{~nm}$ ), small samples $(c .50 \mu \mathrm{l})$ were removed into micro-centrifuge tubes containing $1 \mathrm{ml}$ of a washed suspension of horse red blood cells $2 \% \mathrm{v} / \mathrm{v}$ in saline with and without $20 \mathrm{~mm}$ $\mathrm{CaCl}_{2}$. After the tube contents had been mixed by inversion, the tubes were incubated with gentle agitation in a water bath at $37^{\circ} \mathrm{C}$ for $15 \mathrm{~min}$. The tubes were then centrifuged at $11600 \mathrm{~g}$ for $30 \mathrm{~s}$ to pellet the red blood cells and bacteria. The amount of free haemoglobin in the supernate (measured by its absorbance at $540 \mathrm{~nm}$ against a blank of water) indicated the degree of haemolytic activity.

\section{Results}

\section{The haemolysins of $M$. morgani strains}

A total of 32 strains of $M$. morgani belonging to 31 different bacteriocin $\mathrm{P} / \mathrm{S}$ types was examined. Most (29) strains were isolated from clinical specimens in Dundee ( 22 from faeces, 3 from urine, 3 from wounds, and 1 from CSF). The remaining three strains were known haemolytic strains isolated from clinical specimens in London (1) and Hungary (2). Of the 32 strains, $18(56 \%)$ were haemolytic on blood agar, the colonies being surrounded by a narrow $(<0.5 \mathrm{~mm})$ zone of haemolysis. Pure subcultures of two strains produced some colonies with narrow zones and others with wider $(1 \mathrm{~mm})$ haemolytic zones. This was not altered by repeated subculture of each haemolytic colony type.

Only strains haemolytic on blood agar produced haemolysins in broth. Haemolysin production was unrelated to the source of the strain; the proportion of haemolytic strains from one source-faeces $(45 \%$ haemolytic) - was not significantly different from that of haemolytic strains in the group as a whole. The ability to produce haemolysin was also unrelated to the bacteriocin type of the strain. Both haemolytic and non-haemolytic strains were found among those with the same bacteriocin sensitivity pattern and of the two strains sharing the same bacteriocin $\mathbf{P} / \mathrm{S}$ pattern, one was haemolytic and the other was not.

The kinetics of haemolysin production in $\mathrm{BHI}$ were examined in four strains selected at random from those found to be haemolytic on blood agar and sensitive to $25 \mu \mathrm{g}$ of chloramphenicol. A typical result is shown in fig. 1. For each strain, haemolysin activity was first detected in the early phase of logarithmic growth and it reached a peak toward the end of logarithmic growth. Thereafter, haemolytic activity declined and eventually ceased to be detectable. The decline in haemolytic activity is unlikely to be the result of the synthesis at this stage of a protein able to degrade or inhibit haemolysins because, when chloramphenicol was added to 


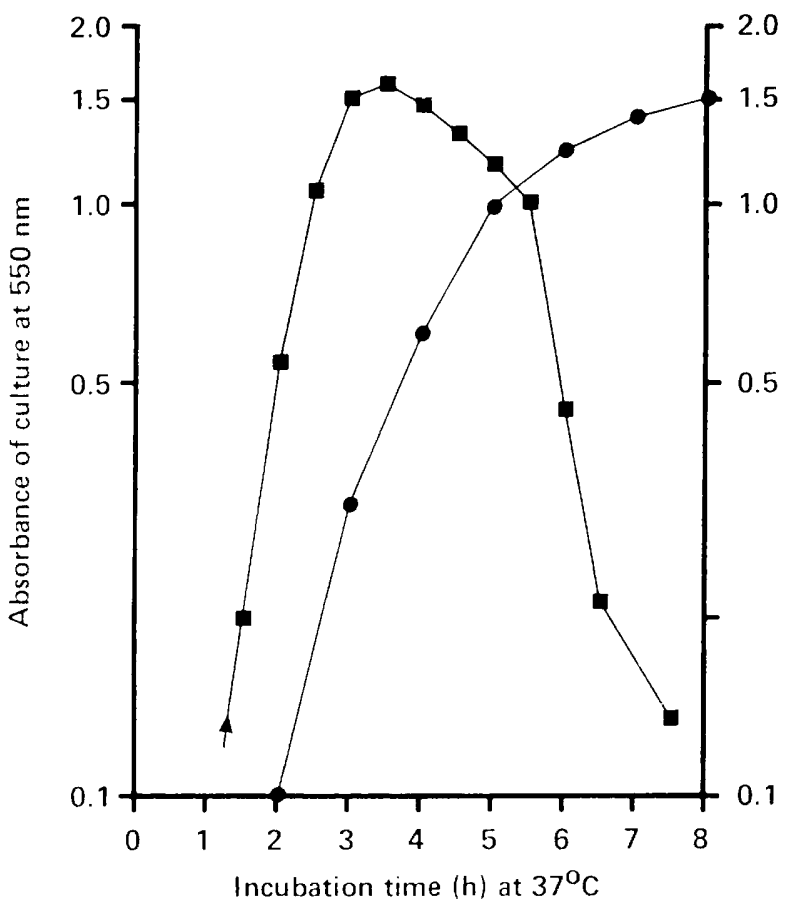

Fig. 1. The kinetics of the development of calcium-dependent haemolytic activity ( $\square$ ) by $M$. morgani strain 227 and its growth (O) in broth

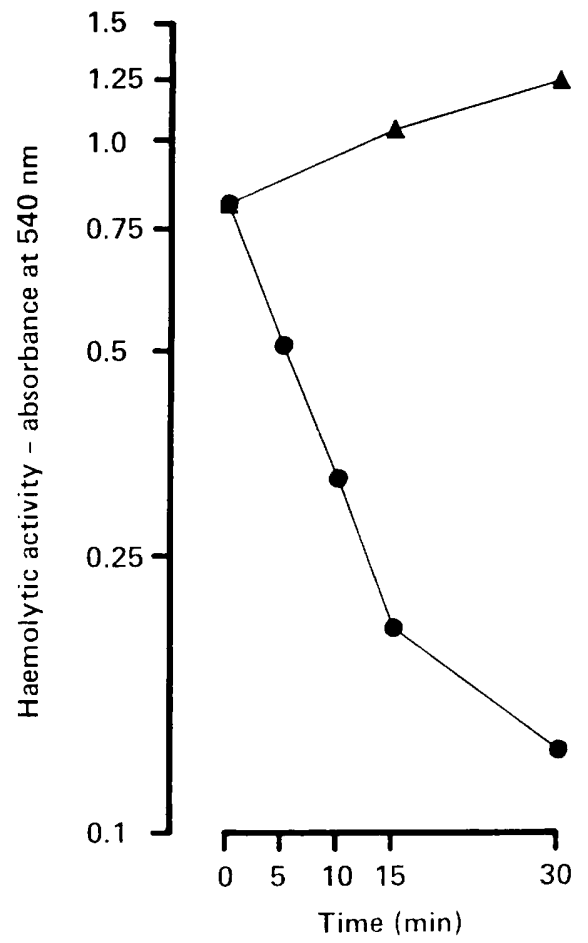

Fig. 2. The effect of the addition of chloramphenicol to a growing culture of $M$. morgani strain 227 on the calcium-dependent haemolytic activity of the cells; (A) untreated culture, (O) culture to which chloramphenicol $25 \mu \mathrm{g} / \mathrm{ml}$ was added at time 0 . culture samples with high haemolytic activity to prevent synthesis of this protein, instead of haemolytic activity being maintained unchanged, the activity rapidly declined (fig. 2). It appeared, therefore, that $M$. morgani haemolysins were extremely unstable with an active half-life of about $10 \mathrm{~min}$ and, through strict regulation, appeared to be synthesised only over a short period during logarithmic growth.

All but one of the haemolytic $M$. morgani strains formed haemolysins which required $\mathrm{Ca}^{2+}$ ions for lytic activity. $\mathrm{CaCl}_{2}$ was added to the assay system to give a final concentration of $20 \mathrm{~mm}$ but experiments showed that the amount could be reduced to $2.5 \mathrm{~mm}$ without effect on the amount of haemolysis. The exceptional strain produced, over a short period, a small amount of a calciumindependent haemolysin a few hours after the peak of calcium-dependent haemolytic activity. $M$. morgani haemolysins were heat-labile; samples taken at the height of haemolysin production did not retain activity after being heated for $5 \mathrm{~min}$ at $100^{\circ} \mathrm{C}$.

\section{The haemolysins of $P$. mirabilis strains}

Of the 32 strains of $P$. mirabilis examined, 26 strains were from five British cities, two were from Germany and four were from Sweden. At least 11 different $\mathrm{O}$-antigenic types and 27 bacteriocin $(\mathrm{P} /$ S) types were represented. Fifteen strains were from patients with urinary-tract infections, 10 strains were from faeces (four from healthy people), two strains were from sputum and there were single isolates from a brain abscess, a ureteric stone, a kidney stone, a vaginal infection and a chronic infection of the middle ear.

None of the strains formed haemolytic zones on horse blood agar within $16 \mathrm{~h}$ at $37^{\circ} \mathrm{C}$. However, after longer incubation and particularly where many colonies were crowded together, discolouration of the medium was followed eventually by a clear wide zone round the colonies. This was not ascribed to haemolysin activity.

Although none of the strains was considered to be haemolytic on blood agar, $30(94 \%)$ produced haemolysins in liquid medium. The two non- 


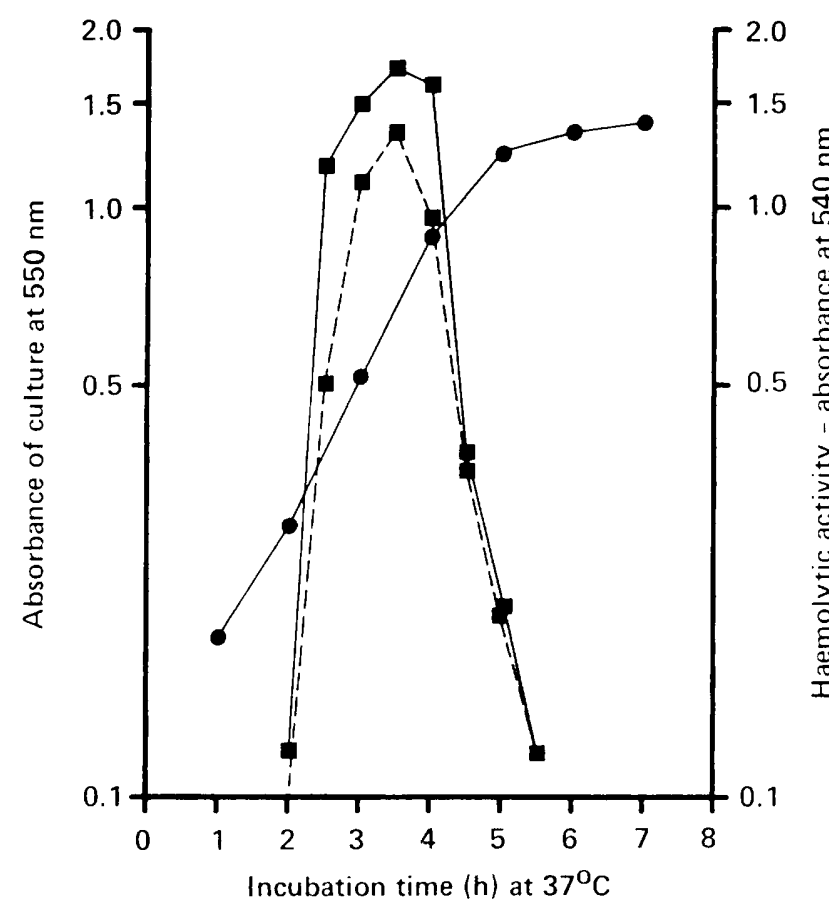

Fig. 3. The kinetics of development of haemolytic activity ( $\square$ ) by $P$. mirabilis strain U6450 and its growth (O) in broth. Haemolytic activity was measured in the presence (- $\longrightarrow$ ) and absence (--) of calcium.

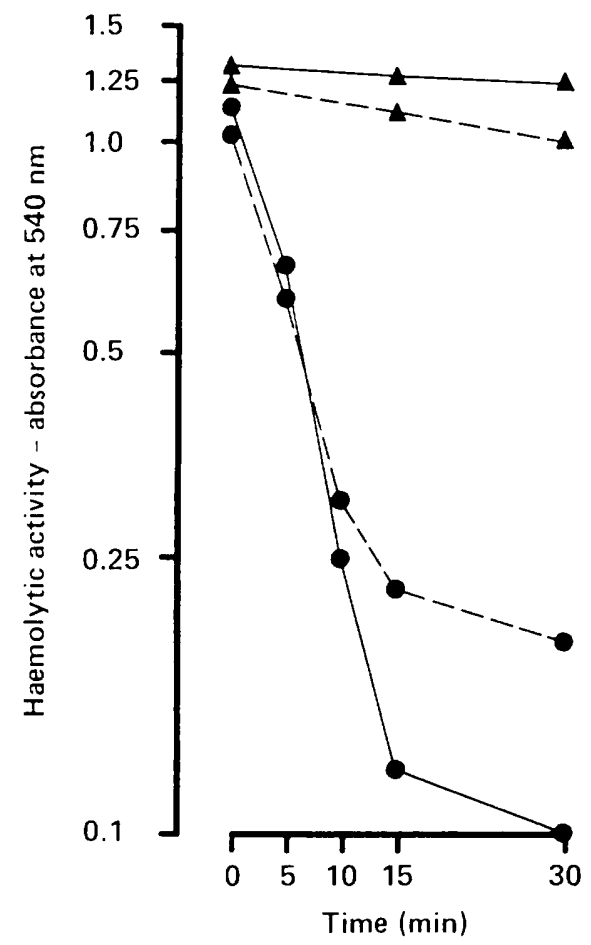

Fig. 4. The effect of the addition of chloramphenicol to a growing culture of $P$. mirabilis strain U6450 on the haemolytic activity of the cells when measured in the presence (and absence (---) of calcium; $(\mathbf{\Lambda})$ untreated culture, $(\mathbf{)}$ culture to which chloramphenicol $25 \mu \mathrm{g} / \mathrm{ml}$ was added at time 0 . haemolytic strains were from the urine and ureteric stone of unrelated people and one of these strains had the same $\mathbf{P} / \mathrm{S}$ type as a haemolytic strain. It appeared, therefore, that the ability to produce haemolysin in liquid medium was a feature unconnected with $\mathrm{P} / \mathrm{S}$ type, O-antigenic type, or source of strain and was characteristic of almost all strains of $P$. mirabilis.

The kinetics of haemolysin production were examined in four of the haemolytic strains. A typical result is shown in fig. 3. Haemolytic activity was first detected in early logarithmic growth and it reached a peak in mid-to-late log-phase growth. Thereafter, there was a rapid, steady decline in activity. The kinetics of haemolysin production were the same whether or not calcium was present although the amount of haemolysis was often a little greater in the presence of calcium. The rapid decline in haemolytic activity was found in the presence of chloramphenicol regardless of whether or not calcium was present. This was believed to be due to the instability in the cultures of the haemolysin which had a half-life of activity of about 5 min (fig. 4).

$P$. mirabilis haemolysins from cultures sampled at maximum haemolytic activity were cell associated, because culture filtrates from a $0 \cdot 22-\mu \mathrm{m}$ membrane filter had no activity. However, $50 \%$ or more of the haemolytic activity was detected after culture samples had been heated for $5 \mathrm{~min}$ in a boiling water bath. In view of the short half-life of the haemolysin, this finding was surprising. The heat treatment may cause release of internal haemolysin or binding of haemolysin to media or cell components which give it stability. The absence of calcium may also stabilise the haemolysin (see Discussion). Examination of mid-log-phase culture samples of the other 26 haemolytic strains showed that all produced haemolysins with the same properties. 
The haemolysins of $P$. vulgaris strains

A total of 31 strains of $P$. vulgaris, 19 of biotype 2 and 12 of biotype 3 , was examined for haemolysin production. The strains were from England, Scotland and Sweden and represented 18 different $P / S$ types; 19 strains were from urine, 8 from faeces, 3 from wounds and one was from an ear infection. On blood agar, $15(48 \%)$ strains formed haemolysins; 10 strains produced narrow zones of haemolysis ( $<1 \mathrm{~mm}$ from the colony edge) and 5 strains produced wide zones $(1-2 \mathrm{~mm}$ from the colony edge). There was no association between biotype and haemolysis, whether small or wide zone; 8 of $19(42 \%)$ biotype- 2 strains and 7 of $12(58 \%)$ biotype3 strains were haemolytic and both biotypes were found among strains producing large haemolytic zones. Only 2 of the $8(25 \%)$ urinary strains but 9 of the $19(47 \%)$ faecal stains were haemolytic on blood agar; 3 of the 4 strains from infected sources other than faeces and urine produced large haemolytic zones whereas among the remaining 27 strains from urine and faeces, only 2 produced large haemolytic zones.

Examination of strains in mid-to-late log-phase growth for ability to produce haemolysin in BHI when activity was assayed in the presence or absence of calcium ( $20 \mathrm{mM})$ revealed that $26(84 \%)$ strains produced haemolysins. Again, there was no association with the biotype of the strain-16 of 19 $(84 \%)$ biotype- 2 and 10 of $12(83 \%)$ biotype- 3 strains were haemolytic - or with the P/S type-haemolytic and non-haemolytic strains were found among those with the same $\mathrm{P} / \mathrm{S}$ type. Of the five strains unable to produce haemolysin in liquid or on solid media, three were from urine and two from faeces.

The kinetics of production and the properties of the haemolysins formed were first examined with two strains that produced haemolysin in BHI but not on blood agar and with two strains that produced haemolysin in BHI and formed narrow zones on blood agar. The kinetics of haemolysin production in BHI by all four strains were very similar and resembled those found with $P$. mirabilis strains (fig. 3). For all four strains, haemolytic activity was first detected in early, and reached a maximum in mid-to-late, log-phase growth. Thereafter, there was a rapid decline in activity. Whether or not calcium was present, the kinetics of development of haemolytic activity were synchronous and the amounts of red-cell lysis virtually identical. This suggested that only a single type of haemolysin, independent of the need for calcium for activity, was being formed. The properties of the haemolysin from all four strains were similar to those of $P$. mirabilis haemolysin; it was unstable with a halflife of activity of about $5 \mathrm{~min}$ in the presence of chloramphenicol (fig. 5-strain F34393) and 50\% or more haemolytic activity remained after cultures were heated for $5 \mathrm{~min}$ in a boiling water bath.

The kinetics of production and the properties of the haemolysins formed in BHI by the five strains that gave large haemolytic zones on blood agar were determined in the presence and absence of calcium. All five strains produced, very rapidly, large amounts of a calcium-dependent haemolysin (fig. 6); culture samples taken after incubation for $1 \mathrm{~h}$ caused lysis of nearly all red cells in the assay system when calcium was present but failed to cause lysis in its absence. Probably because the haemolysin was formed in large amounts, its activity persisted longer than that of the other $P$. vulgaris haemolysins and did not begin to decline until the stationary phase of growth.

All five strains also produced, at a later stage, smaller amounts of a $\mathrm{Ca}^{2+}$-independent haemolysin (fig. 6). The properties of the two haemolysins were compared. By assaying in the presence of $\mathrm{Ca}^{2+}(20 \mathrm{mM})$ culture samples taken within $1 \mathrm{~h}$ of inoculation, which was before formation of the calcium-independent haemolysin commenced, it was shown that for all five strains the activity of the calcium-dependent haemolysin was unstable, with an active half-life of about $5 \mathrm{~min}$ in the presence of chloramphenicol (fig. 5-strain 76362/79), and heat labile - no activity remained after $5 \mathrm{~min}$ in a boiling water bath. The half-life and heat stability of the $\mathrm{Ca}^{2+}$ independent haemolysins were determined from culture samples taken after incubation for $4 \mathrm{~h}$ and assayed in the absence of calcium. These haemolysins shared all the properties of the calciumdependent haemolysin but $50 \%$ or more haemolytic activity remained after cultures were heated for 5 min in a boiling water bath. This haemolysin appeared to be the same as the calcium-independent haemolysin produced by the $P$. vulgaris strains that did not form large haemolytic zones on blood agar.

\section{The haemolysins of $P$. penneri strains}

The 29 strains examined all gave reactions typical for $P$. penneri but maltose fermentation with some strains was delayed for as much as 5-7 days. All the strains were isolated from faeces of patients with (19 strains) and without (10 strains) diarrhoea, but only three different $\mathrm{P} / \mathrm{S}$ types were represented. All the strains were haemolytic on blood agar. One group of six strains, which included all those fermenting maltose within 1 day and all of the $\mathrm{H}_{2} \mathrm{~S}$ producing strains, formed colonies which were 


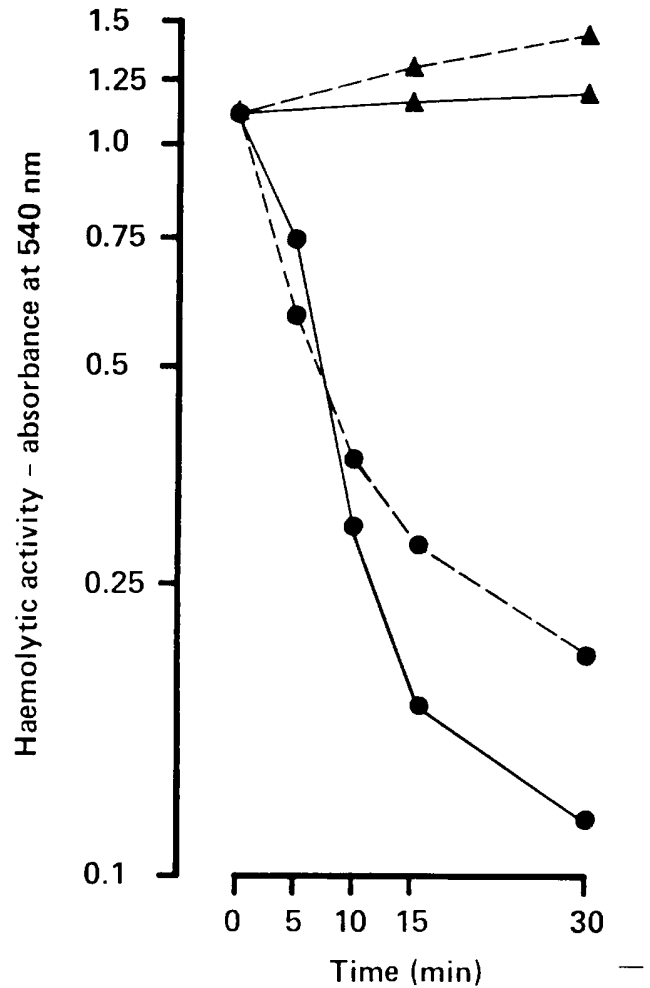

Fig. 5. The effect of the addition of chloramphenicol to growing cultures of $P$. vulgaris strains on the haemolytic activity of the calcium-independent haemolysin of strain F 34393 (-) and the calcium dependent haemolysin of strain $76362 / 79(---)$; ( $\mathbf{\Lambda})$ untreated cultures, (O) cultures in which chloramphenicol $25 \mu \mathrm{g} /$ $\mathrm{ml}$ was added at time 0 .

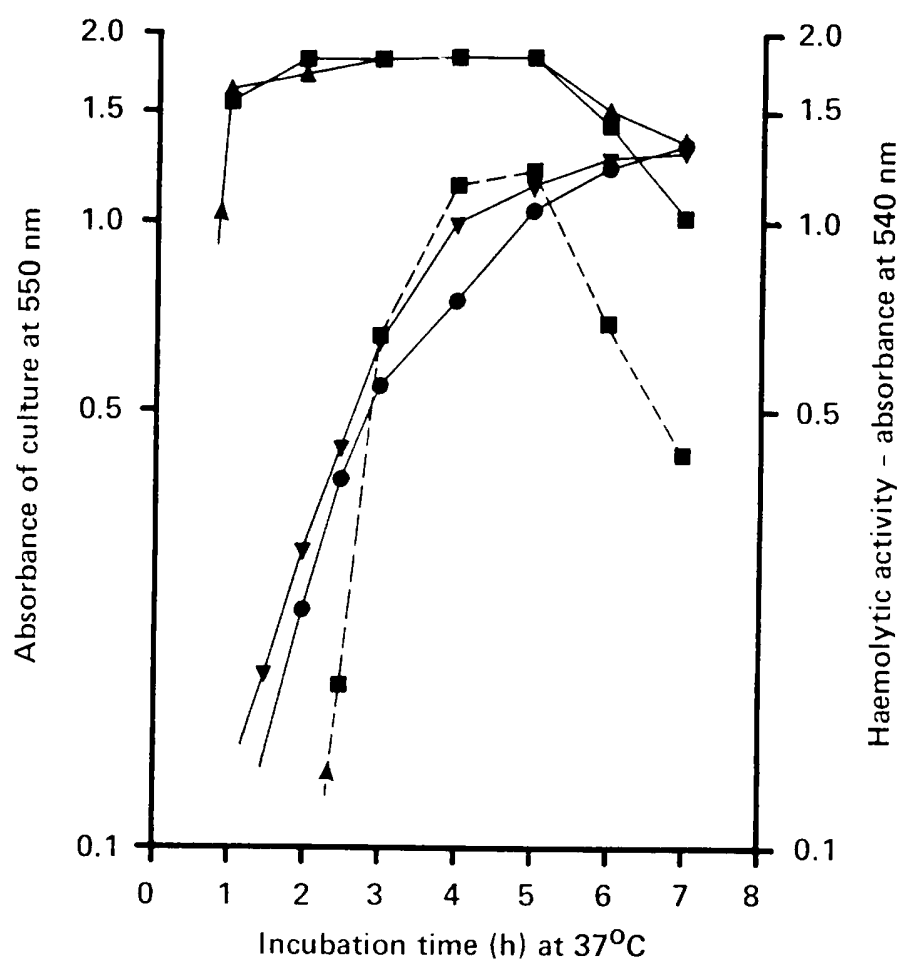

Fig. 6. The kinetics of development of the haemolytic activity of $P$. vulgaris strains $76362 / 79(\mathbf{A})$ and PR3 ( $\mathbf{a}$ ) and their growth in broth $(\nabla$, respectively). Haemolytic activity was measured in the presence (-) and absence (--- ) of calcium. surrounded by wide (1-2 mm) zones of haemolysis. The remaining group of 23 strains of the same $P / S$ type all formed narrow $(0.5 \mathrm{~mm})$ haemolytic zones.

The kinetics of production and the properties of the haemolysins formed by two strains of different $\mathrm{P} / \mathrm{S}$ type that formed wide haemolytic zones, and two strains of the same $\mathrm{P} / \mathrm{S}$ types that formed narrow haemolytic zones were examined in $\mathrm{BHI}$ broth, in the presence and absence of calcium. All four strains showed maximum haemolytic activity in mid-to-late log-phase growth. The two strains forming wide haemolytic zones on blood agar produced, within the first $2 \mathrm{~h}$ of incubation, large amounts of a highly active calcium-dependent haemolysin. The activity of this haemolysin was maintained over a long period and even into the stationary growth phase before there was a slow decline in its activity (fig. 7-strain H 1360). These strains also produced, at a slower rate, smaller amounts of a less active, calcium-independent haemolysin. The properties of the calcium-dependent haemolysin were determined in samples taken after incubation for $2 \mathrm{~h}$ and before the formation of the calcium-independent haemolysin. The calciumdependent haemolysin resembled the calciumdependent haemolysin of $P$. vulgaris strains in being unstable and heat labile.

Each one of the two strains that formed narrow haemolytic zones on blood agar produced, in BHI, what appeared to be a single type of haemolysin that was calcium independent, because the kinetics of development of haemolytic activity were synchronous and the amount of cell lysis virtually identical whether measured in the presence or absence of calcium (fig. 7-strain H 1209). The activity of this haemolysin declined at a faster rate than that of the calcium-dependent haemolysin. It had the same properties as the calcium-dependent haemolysin but was resistant to heating in a boiling water bath for $5 \mathrm{~min}$. 


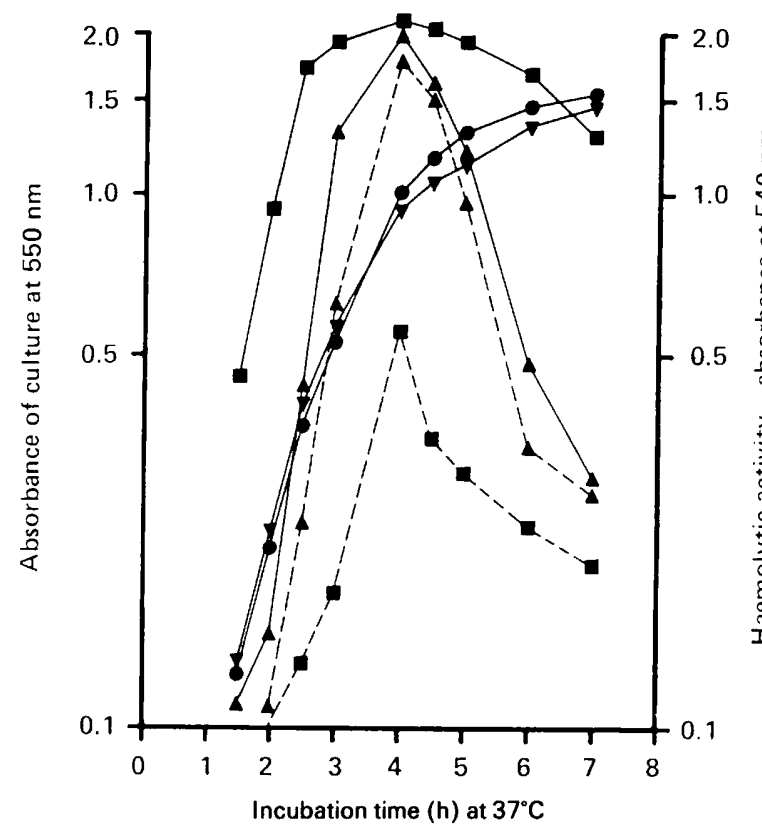

Fig. 7. The kinetics of development of the haemolytic activity of $P$. penneri strains $\mathrm{H} 1360(\square)$ and $\mathrm{H} 1209(\mathbf{\Delta})$ and their growth in broth $(\mathcal{O}, \nabla$ respectively). Haemolytic activity was measured in the presence (- $\longrightarrow$ ) and absence (---) of calcium.

\section{Examination of strains of Providencia spp. for haemolysin production}

A total of 74 strains representing four species of Providencia and six biotypes was examined. Twenty-nine strains gave biochemical reactions of Prov. rettgeri; 15 of these were from clinical specimens from Dundee -9 from faeces and 6 from urine; the other 14 strains were from Canada and represented 14 distinct $\mathrm{O}$-antigenic types. Twenty strains of Prov. alcalifaciens were examined; 10 were of biotype 1 (aerogenic) and 10 of biotype 2 (anaerogenic), and all were isolated from faeces in Dundee. Six strains (5 anaerogenic) that gave reactions of Prov. rustigiani were isolated from faeces in Dundee. The remaining 19 strains were Prov. stuarti-18 of biotype 5 and one of biotype 4 . These were isolated from clinical specimens in Dundee-13 from urine, 5 from faeces and one from a wound.

None of the 74 strains of Providencia species produced haemolysin either on blood agar or in BHI. The latter analysis was made in the presence and absence of calcium with increased samples of $100 \mu \mathrm{l}$ which were taken during both the mid- and late-logarithmic growth phases.

\section{Discussion}

Production of haemolysin was very common in two of the three genera of the Proteeae. It was a stable characteristic of most strains of Proteus irrespective of their species, type or origin. Thus, all $P$. penneri strains, $94 \%$ of $P$. mirabilis strains and $84 \%$ of $P$. vulgaris strains were haemolytic. Haemolysin production was also common among $M$. morgani, $56 \%$ of strains being haemolytic, a higher percentage than the $33 \%$ found by Rauss (1936). However, among 74 strains of six biotypes of Providencia spp. examined, not one haemolytic strain was found.

The haemolysins produced by strains of Proteus and Morganella appeared to be of two types. One was calcium independent and was produced alone by most strains of Proteus spp. The other type was calcium dependent and was produced almost exclusively by haemolytic $M$. morgani strains and a few strains of $P$. vulgaris and $P$. penneri which also produced the calcium-independent type of haemolysin. Moreover, the haemolysins of the different species varied in their response to heat. However, since we have recently shown (Koranakis et al., 1987) that DNA from some of the haemolytic strains of $P$. mirabilis, $P$. vulgaris and $M$. morgani used in this study hybridised with a DNA probe of the $E$. coli haemolysin structural gene ( $h l y \mathrm{~A}$ ), these differences have to be regarded as being only superficial. They may arise from differences in complexes formed between haemolysin and lipopolysaccharides of the various species and between haemolysin and broth components.

The haemolytic activity of $E$. coli strains is generally calcium dependent (Bamforth and Dudgeon, 1952; Snyder and Zwadyk, 1969; Short and Kurtz, 1971; Rennie et al., 1974) and heat labile (Lovell and Rees, 1960; Smith, 1963; Snyder and Koch, 1966; Rennie and Arbuthnott, 1974; Jorgensen et al., 1976). Since, however, the heat lability of the haemolysin is influenced by calcium-being more stable in its absence (Snyder and Koch, 1966) - it is easy to understand why the calciumindependent haemolysin of Proteus spp. whose activity was determined in the absence of calcium appeared to be heat resistant. Calcium is also known to shorten the lag phase of the haemolytic reaction of $E$. coli by activating haemolysin in some way (Rennie et al., 1974). This could be the explanation for the slightly greater haemolytic activity often observed when calcium-independent haemolysins were assayed in the presence of calcium. The apparent late production by some strains of $P$. vulgaris and $P$. penneri of smaller 
amounts of a second type of haemolytic activity, now calcium independent, may simply be another manifestation of delayed haemolysin activation in the absence of calcium.

Differences were also observed in the activity on blood agar of the haemolysins produced by the different bacterial species. None of the strains of $P$. mirabilis producing the calcium-independent type of haemolysin formed zones on blood agar, whereas $58 \%$ of the $P$. vulgaris strains forming the same haemolysin did. The calcium-dependent haemolysin produced by $M$. morgani strains formed narrow haemolytic zones but when produced by $P$. vulgaris and $P$. penneri strains formed large zones. These differences indicate variation between the species in the secretion of haemolysin.

In $E$. coli, the production and release of haemolysin is determined by four contiguous genes. Genes hly $\mathrm{A}$ and hly $\mathrm{C}$ code for the synthesis of active haemolysin and genes $h l y \mathbf{B}$ and $h l y \mathrm{D}$ code for its active transport through both cell membranes. Complete haemolysin transport requires the formation of the products of both $B$ and $D$ genes. Loss of the hly D gene results in incomplete transport (Hacker and Hughes, 1985). Koranakis et al. (1987) have shown that DNA from haemolytic strains of species within the Proteeae share homology with the hly A, B and C genes of E. coli. Homology with the hly D genes was only found in DNA from strains of $P$. vulgaris and $M$. morgani producing cell-free haemolysin. No homology was found with DNA from haemolytic $P$. mirabilis strains. The lack of the hly $\mathrm{D}$ gene in P. mirabilis strains supports our findings that haemolysin in this organism is incompletely secreted and is cell-associated, and, because of this, haemolytic zones are not seen on blood agar.

Among the Proteeae, haemolysin production has

\section{REFERENCES}

Bamforth J, Dudgeon J A 1952 The haemolytic activity of Bact. coli. Journal of Pathology and Bacteriology 64: 751-761.

Bergström T 1972 Sex differences in childhood urinary tract infection. Archives of Diseases of Childhood 47: 227-232.

Brooks H J L, O'Grady F, McSherry M A, Cattell W R 1980 Uropathogenic properties of Escherichia coli in recurrent urinary-tract infection. Journal of Medical Microbiology 13: $57-68$.

Cooke E M, Ewins S P 1975 Properties of strains of Escherichia coli isolated from a variety of sources. Journal of Medical Microbiology 8: 107-111.

Dudgeon L S, Wordley E, Bawtree F 1921 On Bacillus coli infections of the urinary tract, especially in relation to haemolytic organisms. Journal of Hygiene 20 : 137-164.

Emödy L, Vörös S, Pál T 1982 Alpha-haemolysin, a possible been shown to be associated with the virulence of $M$. morgani (Emody et al., 1982) and P. mirabilis (Peerbooms et al., 1983) for mice and with the invasion of mammalian cells by $P$. mirabilis (Peerbooms et al., 1984). However, the relative importance of haemolysin production as a virulence factor for $P$. mirabilis in infections of the renal tract in man, compared to that of other known virulence mechanisms, remains to be determined.

Because the haemolysin produced by haemolytic strains of the Proteeae is related to that known to contribute to the virulence of $E$. coli for the urinary tract, the finding that nearly all strains of Proteus formed the haemolysin suggests that it might also be a factor contributing to the virulence of the species of this genus for the urinary tract. The rare association of $P$. vulgaris (and probably $P$. penneri) strains with urinary tract infection (Senior, 1979) must, therefore, be the outcome of their rare occurrence in faeces (Senior and Leslie, 1986) rather than their lacking this virulence factor.

Strains of $M$. morgani are rarely associated with urinary-tract infections (Senior, 1979). This has been explained on the basis of their slow growth rate in urine and their inability to make urine alkaline rapidly (Senior, 1983). The finding that approximately half these organisms are unable to produce haemolysin suggests that such strains are at an even greater disadvantage as potential urinary-tract pathogens. Strains of Providencia spp. are relatively rarely the primary cause of urinarytract infections. The finding that they do not form haemolysins supports the view that these organisms have a low virulence for the urinary tract.

We are grateful to Drs P. M. Hawkey, P. Larsson, R. Maskell, H. E. Müller, J. L. Penner and G. Sweeney for the gift of some of the strains. virulence factor in Proteus morganii. FEMS Microbiology Letters 13: 329-331.

Green C P, Thomas V L 1981 Hemagglutination of human type O erythrocytes, hemolysin production, and serogrouping of Escherichia coli isolates from patients with acute pyelonephritis, cystitis, and asymptomatic bacteriuria. Infection and Immunity 31 : 309-315.

Hacker J, Schroter G, Schrettenbrunner A, Hughes C, Goebel W $1983 a$ Hemolytic Escherichia coli strains in the human fecal flora as potential urinary pathogens. Zentralblatt für Bakteriologie, Mikrobiologie und Hygiene, 1 Abteilung Originale A 254: 370-378.

Hacker J, Hughes C, Hof H, Goebel W $1983 b$ Cloned hemolysin genes from Escherichia coli that cause urinary tract infection determine different levels of toxicity in mice. Infection and Immunity 42: 57-63.

Hacker J, Hughes C 1985 Genetics of Escherichia coli hemolysin. 
Current Topics in Microbiology and Immunology, 118: 138162.

Hallett R J, Pead L, Maskell R 1976 Urinary infection in boys. A three year prospective study. Lancet 2: 1107-1110.

Hickman F W, Steigerwalt A G, Farmer J J, Brenner D J 1982 Identification of Proteus penneri sp. nov. formerly known as Proteus vulgaris indole negative or as Proteus vulgaris biogroup 1. Journal of Clinical Microbiology 15: 1097-1102.

Hickman-Brenner F W, Farmer J J, Steigerwalt A G, Brenner D J 1983 Providencia rustigianii: a new species in the family Enterobacteriaceae formerly known as Providencia alcalifaciens biogroup 3. Journal of Clinical Microbiology 17: $1057-1060$.

Hughes C, Hacker J, Roberts A, Goebel W 1983 Hemolysin production as a virulence marker in symptomatic and asymptomatic urinary tract infections caused by Escherichia coli. Infection and Immunity 39: 546-551.

Jörgensen S E, Short E C, Kurtz H J, Mussen H K, Wu G K 1976 Studies on the origin of the $\alpha$-haemolysin produced by Escherichia coli. Journal of Medical Microbiology 9: 173-189.

Khan A J, Ubriani R S, Bombach E, Agbayani M M, Ratner $H$, Evans H E 1978 Initial urinary tract infection caused by Proteus mirabilis in infancy and childhood. Journal of Pediatrics 93: 791-793.

Koronakis V, Cross M, Senior B, Koronakis E, Hughes C 1987 The secreted hemolysins of Proteus mirabilis, Proteus vulgaris and Morganella morganii are genetically related to each other and to the alpha-hemolysin of Escherichia coli. Journal of Bacteriology 169: 1509-1515.

Kotelko K, Kaca W, Rozalski A, Deka M 1983 Some biological features of Proteus Bacilli. 2. Haemolytic activities of Proteus mirabilis and Proteus vulgaris strains. Acta Microbiologica Polonica 32: 345-351.

Larsson P, Olling S $1977 \mathrm{O}$ antigen distribution and sensitivity to the bactericidal effect of normal human serum of Proteus strains from clinical specimens. Medical Microbiology and Immunology 163: 77-82.

Lovell R, Rees T A 1960 A filterable haemolysin from Escherichia coli. Nature 188: 755-756.

Peerbooms P G H, Verweij A M J J, MacLaren D M 1983 Investigation of the haemolytic activity of Proteus mirabilis strains. Antonie van Leeuwenhoek 49: 1-11.

Peerbooms P G H, Verweij A M J J, MacLaren D M 1984 Vero cell invasiveness of Proteus mirabilis. Infection and Immunity 43: $1068-1071$.
Rauss K F 1936 The systematic position of Morgan's Bacillus. Journal of Pathology and Bacteriology 42: 183-192.

Rennie R P, Arbuthnott J P 1974 Partial characterisation of Escherichia coli haemolysin. Journal of Medical Microbiology 7: 179-188.

Rennie R P, Freer J H, Arbuthnott J P 1974 The kinetics of erythrocyte lysis by Escherichia coli haemolysin. Journal of Medical Microbiology 7: 189-195.

Senior B W 1977 Typing of Proteus strains by proticine production and sensitivity. Journal of Medical Microbiology 10: $7-17$.

Senior B W 1979 The special affinity of particular types of Proteus mirabilis for the urinary tract. Journal of Medical Microbiology 12: 1-8.

Senior B W 1983 Proteus morgani is less frequently associated with urinary tract infections than Proteus mirabilis-an explanation. Journal of Medical Microbiology 16: 317-322.

Senior B W 1987 The typing of Morganella morgani by bacteriocin production and sensitivity. Journal of Medical Microbiology 23: 33-39.

Senior B W, Larsson P 1983 A highly discriminatory multityping scheme for Proteus mirabilis and Proteus vulgaris. Journal of Medical Microbiology 16: 193-202.

Senior B W, Leslie D L 1986 Rare occurrence of Proteus vulgaris in faeces: a reason for its rare association with urinary tract infections. Journal of Medical Microbiology 21 : 139-144.

Short E C, Kurtz H J 1971 Properties of the hemolytic activities of Escherichia coli. Infection and Immunity 3: 678-687.

Smith H W 1963 The haemolysins of Escherichia coli. Journal of Pathology and Bacteriology 85: 197-211.

Snyder I S, Koch N A 1966 Production and characteristics of hemolysins of Escherichia coli. Journal of Bacteriology 91 : 763-767.

Snyder I S, Zwadyk P 1969 Some factors affecting production and assay of Escherichia coli haemolysins. Journal of General Microbiology 55: 139-143.

Taylor J F 1928 B. proteus infections. Journal of Pathology and Bacteriology 31 : 897-915.

Welch R A, Dellinger E P, Minshew B, Falkow S 1981 Haemolysin contributes to virulence of extra-intestinal $E$. coli infections. Nature 294: 665-667.

Wenner J J, Rettger L F 1919 Systematic study of the Proteus group of bacteria. Journal of Bacteriology 4: 331-353.

Yacob M 1932 Studies of the genus Proteus. Indian Journal of Medical Research 19: 787-829. 